\title{
Aberrant DNA methylation associated with Alzheimer's disease in the superior temporal gyrus
}

\author{
ZHAN GAO, HONG-JUAN FU, LI-BO ZHAO, ZHUO-YAN SUN, YU-FEI YANG and HONG-YAN ZHU \\ Department of Senile Neurology, Heilongjiang Provincial Hospital, Harbin, Heilongjiang 150036, P.R. China
}

Received June 14, 2017; Accepted September 14, 2017

DOI: $10.3892 /$ etm.2017.5394

\begin{abstract}
Abnormal DNA methylation patterns have been demonstrated to be associated with the pathogenesis of Alzheimer's disease (AD). The present study aimed to identify differential methylation in the superior temporal gyrus (STG) of patients with late-onset AD based on epigenome-wide DNA methylation data by bioinformatics analysis. The genome-wide DNA methylation data in the STG region of 34 patients with late-onset AD and 34 controls without dementia were recruited from the Gene Expression Omnibus database. Through systemic quality control, differentially methylated $\mathrm{CpG}$ sites were determined by the Student's t-test and mean methylation value differences between the two conditions. Hierarchical clustering analysis was applied to assess the classification performance of differentially methylated CpGs. Functional analysis was performed to investigate the biological functions of the genes associated with differentially methylated $\mathrm{CpGs}$. A total of 17,895 differentially methylated $\mathrm{CpG}$ sites were initially identified, including 11,822 hypermethylated CpGs and 6,073 hypomethylated CpGs. Further analysis examined 2,211 differentially methylated CpGs (covering 1,991 genes). AD subjects demonstrated distinctive DNA methylation patterns when compared with the controls, with a classification accuracy value of 1 . Hypermethylation was mainly detected for genes regulating the cell cycle progression, whereas hypomethylation was observed in genes involved in transcription factor binding. The present study demonstrated widespread and distinctive DNA methylation alterations in late-onset AD. Identification of AD-associated epigenetic biomarkers may allow for the development of novel diagnostic and therapeutic targets.
\end{abstract}

Correspondence to: Dr Hong-Yan Zhu, Department of Senile Neurology, Heilongjiang Provincial Hospital, 82 Zhongshan Road, Xiangfang, Harbin, Heilongjiang 150036, P.R. China

E-mail: zhuhongyan1978@163.com

Key words: Alzheimer's disease, DNA methylation, superior temporal gyrus, epigenetic biomarkers

\section{Introduction}

Alzheimer's disease (AD) is a chronic degenerative brain disorder and the leading cause of dementia in the elderly $(1,2)$. $\mathrm{AD}$ is characterized by gradual neuronal death, memory loss and impaired cognitive ability, and contributes to $60-70 \%$ of dementia cases (2). Current advances are able to temporarily improve symptoms, while no effective treatment is available that can cure AD or reverse the progressive course (3). Understanding the risk factors for AD contributes to the investigations on reducing the disease occurrence. The vast majority of patients with AD have late-onset disease, and the susceptible risk is determined by complex mechanisms $(2,4)$.

Previous studies have revealed the roles of genetic, environmental and epigenetic factors in AD risk (4-6). Epigenetics refers to DNA modifications that alter gene expression and phenotype without causing changes to the nucleotide sequences (7). As the best-studied epigenetic modification, DNA methylation is closely associated with several key cellular processes, including cell differentiation, gene regulation and genomic imprinting (8-10). Several lines of evidence has indicated the effect of DNA methylation on the pathogenesis of AD (11-13). Furthermore, the genome-wide methylation profiling technique has been applied for the characterization of methylation patterns across the genome, and has been considered as a reliable tool in identifying methylation differences associated with complex diseases $(13,14)$.

Numerous studies have focused on the identification of specific brain regions that are particularly vulnerable throughout the progression of AD (15-17). Haroutunian et al (18) performed an extensive study on the transcriptional vulnerability involved in the progression of late-onset AD across 15 brain regions, and observed that the superior temporal gyrus (STG) region presented significant transcriptional abnormalities. Thus, the present study focused on the STG region of patients with late-onset AD. Recently, Watson et al (19) performed a genome-wide DNA methylation profiling in the STG region of patients with late-onset $\mathrm{AD}$, and focused on the identification of differentially methylated regions. In the present study, the publicly available DNA methylation data of the study by Watson et al (19) were used to extract AD-associated methylated genes and identify the underlying biological processes impacted by hypomethylated and hypermethylated genes. Furthermore, epigenetic biomarkers in the STG region associated with $\mathrm{AD}$ risk were identified in the current study, and the 
findings may contribute to the development of novel diagnostic and therapeutic targets for late-onset AD.

\section{Materials and methods}

Genome-wide DNA methylation data. The genome-wide DNA methylation data of late-onset $\mathrm{AD}$ were obtained from the Gene Expression Omnibus database (GEO; https://www.ncbi. nlm.nih.gov/geo/), under the GSE76105 accession number (19). The current study was performed using the Illumina Infinium Human Methylation 450 array platform (Illumina, Inc., San Diego, CA, USA). The data analysed from the database included tissue samples obtained from the STG region of 34 patients with confirmed late-onset $\mathrm{AD}$ and 34 controls without dementia. Detailed information of these tissue samples is described in a previous study (19).

Prior to analysis, the raw DNA methylation data were subjected to a rigorous preprocessing procedure following the method developed by Huyn et al (20). Probes were removed from the data according to the following criteria: i) Single nucleotide polymorphisms within $5 \mathrm{bp}$ upstream of the targeted $\mathrm{CpG}$; ii) minimum allelic frequency of $<0.05$; iii) probes on $\mathrm{X}$ and $\mathrm{Y}$ chromosomes; and iv) cross-hybridising probes. Subsequent to applying the exclusion criteria, a total of 424,497 CpGs were selected and used for subsequent analysis. These remaining CpGs were quantile normalized using the lumi package in R software (21) and the beta-mixture quantile method (22).

Differential methylation analysis. In the dataset, the methylation values for individual $\mathrm{CpGs}$ in each sample were expressed as $\beta$-values. $\beta$-value is a quantitative measure of methylation for each $\mathrm{CpG}$ site, ranging between 0 (completely unmethylated site) to 1 (fully methylated site). Initially, the $\beta$-values of individual $\mathrm{CpG}$ sites were obtained in $\mathrm{AD}$ subjects and controls, respectively. Next, the mean $\beta$-value difference of each $\mathrm{CpG}$ between two conditions was calculated, and $\mathrm{CpG}$ sites with an absolute mean $\beta$-value difference of $>0.05$ were included into the study. For each probe, $\beta$-values in $\mathrm{AD}$ subjects were compared against the controls using the Student's t-test. The CpGs with an absolute mean $\beta$-value differences of $>0.05$, as well as $\mathrm{P}<0.05$, were considered to be differentially methylated sites.

Previous studies indicated that methylation alterations rarely exist in genomic regions at the extreme ends of methylation values $(<0.2$ or $>0.8)$, but are preferentially detected in genomic locations at intermediate methylation levels, which is a range associated with active distal regulatory regions, such as enhancers $(20,23)$. Thus, in order to reduce the number of non-variable $\mathrm{CpGs}$ and improve the statistical power of subsequent analyses, the $\mathrm{CpG}$ sites with $\beta$-values of $>0.8$ and $<0.2$ in all samples were removed from the current study. In addition, it is reported that numerous differentially methylated CpGs with a $\beta$-value difference of $<0.2$ may be difficult to reproduce by alternative methodologies or in replication studies (24). Therefore, only CpGs that had a mean $\beta$-value difference of $\geq 0.2$ were retained in the current study.

Hierarchical clustering analysis. Hierarchical clustering is a method of cluster analysis that seeks to build a hierarchy of clusters in data mining and statistics. Theoretically, samples with similar methylation profiles can be clustered together. To assess the classification performance of differentially methylated $\mathrm{CpGs}$, a hierarchical clustering analysis was applied using the Euclidian distance and average linkage criteria (25). Ideally, the samples should be classified into two distinct clusters, namely the AD subjects and controls. In order to assess the classification efficiency, the accuracy was measured, which is a fraction of correctly classified samples over all samples, according to the following formula: Accuracy $=(\mathrm{TP}+\mathrm{TN}) /(\mathrm{TP}+\mathrm{FP}+\mathrm{TN}+\mathrm{FN})$. TP represents the number of positive samples correctly predicted as positive, $\mathrm{TN}$ is the number of negative samples correctly predicted as negative, FP is the number of negative samples incorrectly predicted as positive, and FN represents the number of positive samples incorrectly predicted as negative.

Functional enrichment analysis. To further investigate the biological functions of the genes associated with differentially methylated CpGs, functional enrichment analysis was performed based on Gene Ontology (GO; http://www.geneontology.org) and Kyoto Encyclopedia of Genes and Genomes database (KEGG; http://www.genome.jp/kegg/pathway.html). In addition, enrichment analysis was conducted using the online software Database for Annotation, Visualization and Integrated Discovery (DAVID 6.8; https://david.ncifcrf.gov). Fisher's test was utilized to measure the significance of GO terms and biological pathways. The P-values were adjusted using Benjamini-Hochberg false discovery rate (FDR). Statistically significantly different terms were determined under the criterion of $\mathrm{P}<0.01$.

\section{Results}

Differential methylation analysis. The present study focused on comparing the DNA methylation in the STG region of 34 late-onset AD subjects with that in 34 controls without dementia. Following quality control processing of the DNA methylation data, probes were removed under the filtering criteria described earlier, and methylation data for 424,497 autosomal CpGs in each of the 68 individuals were used in subsequent differential methylation analysis. Under the criteria of absolute mean $\beta$-value differences between AD subjects and controls of $>0.05$, as well as $\mathrm{P}<0.05$, a total of 17,895 differentially methylated $\mathrm{CpG}$ sites covering 8,678 genes were initially identified. Among these differentially methylated CpGs, a total of 11,822 CpGs were hypermethylated and 6,073 $\mathrm{CpGs}$ were hypomethylated. A volcano plot exhibiting the distribution of differentially methylated CpGs is shown in Fig. 1.

Since methylation changes were preferentially detected in genomic regions at intermediate methylation levels, further selection was performed to reduce non-variable CpGs and improve the statistical power. By removing $\mathrm{CpG}$ at the extreme ends of methylation values $(\beta$-values of $>0.8$ and $<0.2$ ), 2,225 differentially methylated CpGs covering 2,001 genes were identified. Furthermore, differentially methylated CpGs with a $\beta$-value difference of $<0.2$ were considered to be difficult to reproduce and excluded. Thus, only differentially methylated CpGs with a mean $\beta$-value difference of $\geq 0.2$ were 


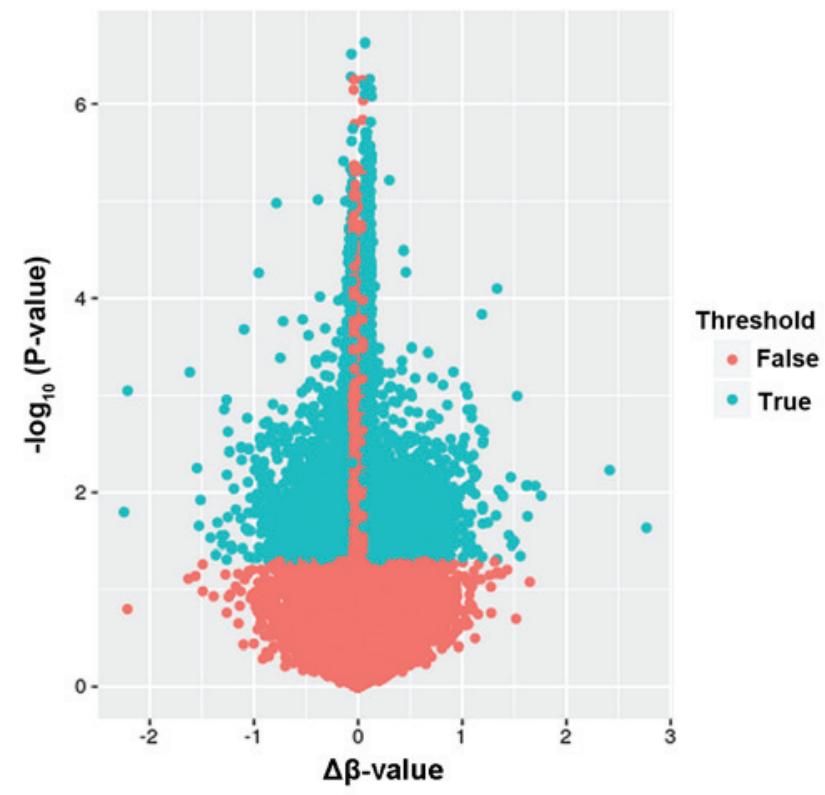

Figure 1. Volcano plot exhibiting the distribution of differentially methylated CpGs in patients with $\mathrm{AD}$ compared with those in control individuals. The $\mathrm{x}$-axis represents the mean methylation differences between AD patients and controls, while the $\mathrm{y}$-axis represents the log transformed P-values. AD, Alzheimer's disease.

extracted, and 2,211 differentially methylated CpGs (covering 1,991 genes) were finally identified.

Hierarchical clustering analysis. Hierarchical clustering was implemented to determine whether the identified differentially methylated $\mathrm{CpGs}$ can be applied to distinguish AD subjects from controls. The results illustrated that these two conditions in the AD and control groups exhibited distinctive DNA methylation patterns (Fig. 2). In addition, it was observed that the samples were classified into two distinct clusters by these differentially methylated CpGs with an accuracy of 1 .

Functional enrichment analysis. GO terms and KEGG pathway enrichment analysis for genes associated with the 2,211 differentially methylated CpGs were performed using the DAVID online tool. Under the criterion of $\mathrm{P}<0.01$, hypermethylated genes were significantly enriched in $10 \mathrm{GO}$ terms, with the top three (ranked according to their P-value) involved in the regulation of the mitotic cell cycle, regulation of the mitotic cell cycle phase transition, and the Wnt signaling pathway (Fig. 3). The hypomethylated genes were significantly enriched in $5 \mathrm{GO}$ terms, with the top one associated with transcription factor binding (Fig. 4). However, KEGG pathway analysis demonstrated that no statistically significant pathways were enriched by both the hypermethylated and hypomethylated genes under an FDR-adjusted P-value of $<0.01$.

\section{Discussion}

Recently, epigenetic modification in diseases has attracted increasing attention from researchers. Late-onset AD results from the interactions of multiple genetic and environmental factors, along with the disruption of epigenetic mechanisms controlling gene expression (26). Abnormal DNA methylation

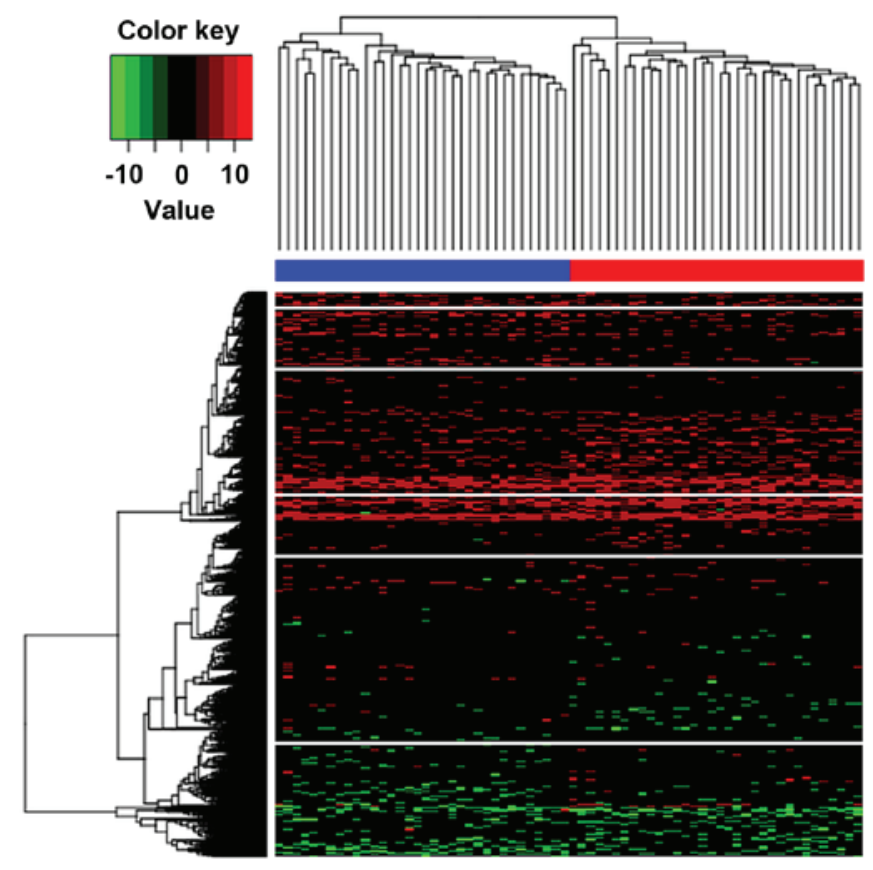

Figure 2. Hierarchical clustering analysis of differentially methylated CpGs between Alzheimer's disease samples and controls. The differentially methylated CpGs were able to distinctly distinguish between the two types of samples.

patterns have been observed to be associated with the pathogenesis of AD. In the present study, the epigenome-wide DNA methylation patterns in the STG of patients with late-onset AD were assessed, and differentially methylated genes and biological functions were identified under the AD condition, which may contribute to the development of novel targets for late-onset $\mathrm{AD}$ diagnosis and therapy.

The present study revealed significant alterations to the DNA methylation profiles in AD, with 2,211 CpGs displaying significantly differential methylation levels. The hierarchical clustering results illustrated that AD subjects exhibited distinctive DNA methylation patterns compared with the controls, which was consistent with the observations of a previous study (19). Initially, the differential methylation identification demonstrated a strong bias to hypermethylated alterations in late-onset $\mathrm{AD}(11,822$ hypermethylated and 6,073 hypomethylated $\mathrm{CpGs}$ ). Given that the greatest risk factor for late-onset $\mathrm{AD}$ is older age (27), it may be hypothesized that CpG methylation increased with age. Previous studies also indicated that $\mathrm{CpG}$ methylation in $\mathrm{AD}$ was significantly associated with age $(13,28)$.

To further examine the functions of genes associated with differentially methylated CpGs, GO and KEGG analysis were performed in the present study. The results revealed that genes associated with hypermethylated $\mathrm{CpGs}$ were enriched in $10 \mathrm{GO}$ terms, 6 of which were cell cycle-associated biological process. The cell cycle involves a series of events that lead to cell division and DNA replication to produce two daughter cells. Cell cycle progression ensures a correct inheritance of epigenetic modifications. A previous study has indicated that epigenetic modifications arising within the cells of an individual are transmitted through multiple cell cycles, which is crucial in maintaining a given chromatin 


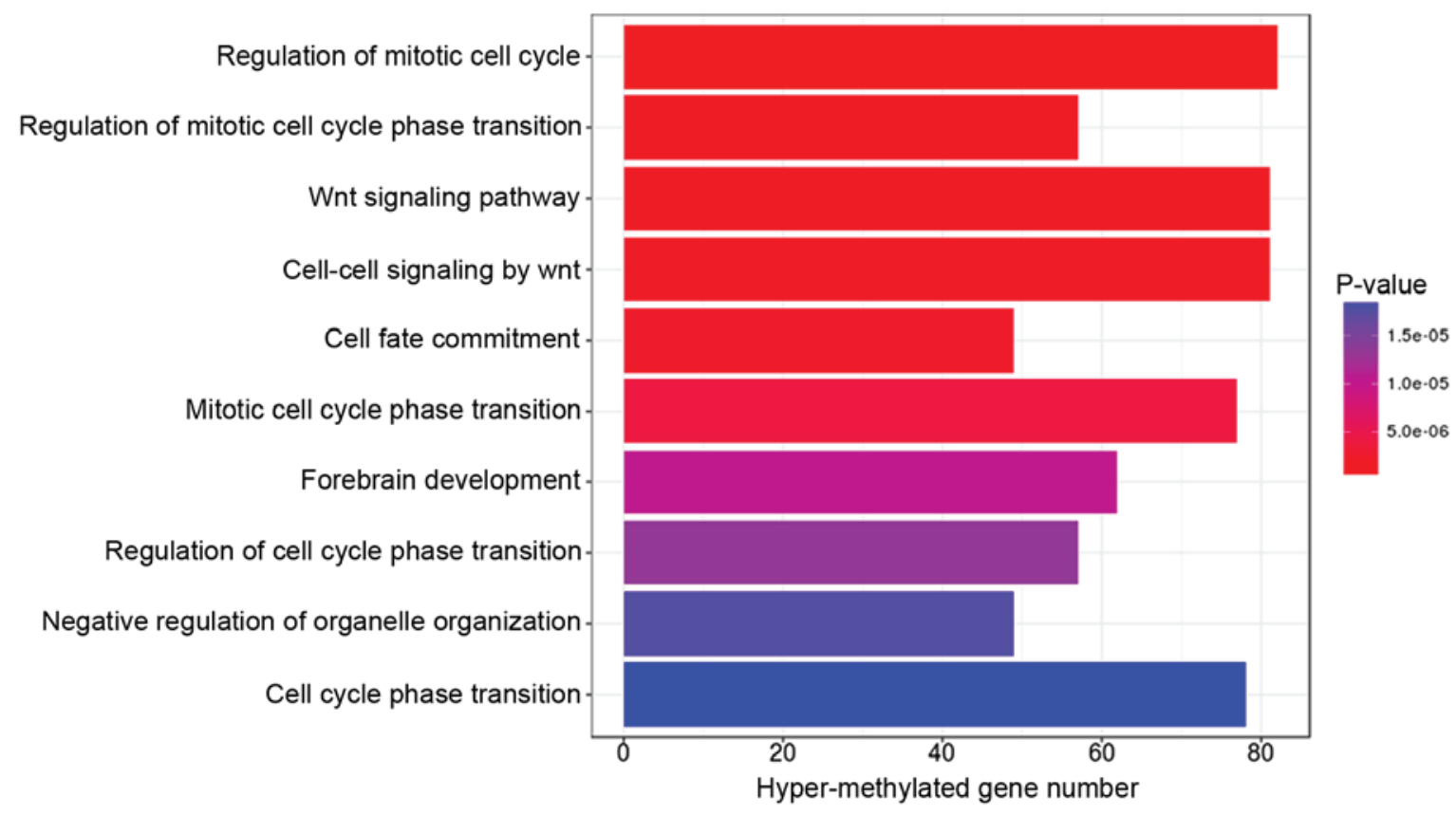

Figure 3. Gene oncology analysis of genes associated with hypermethylated CpGs in Alzheimer's disease subjects.

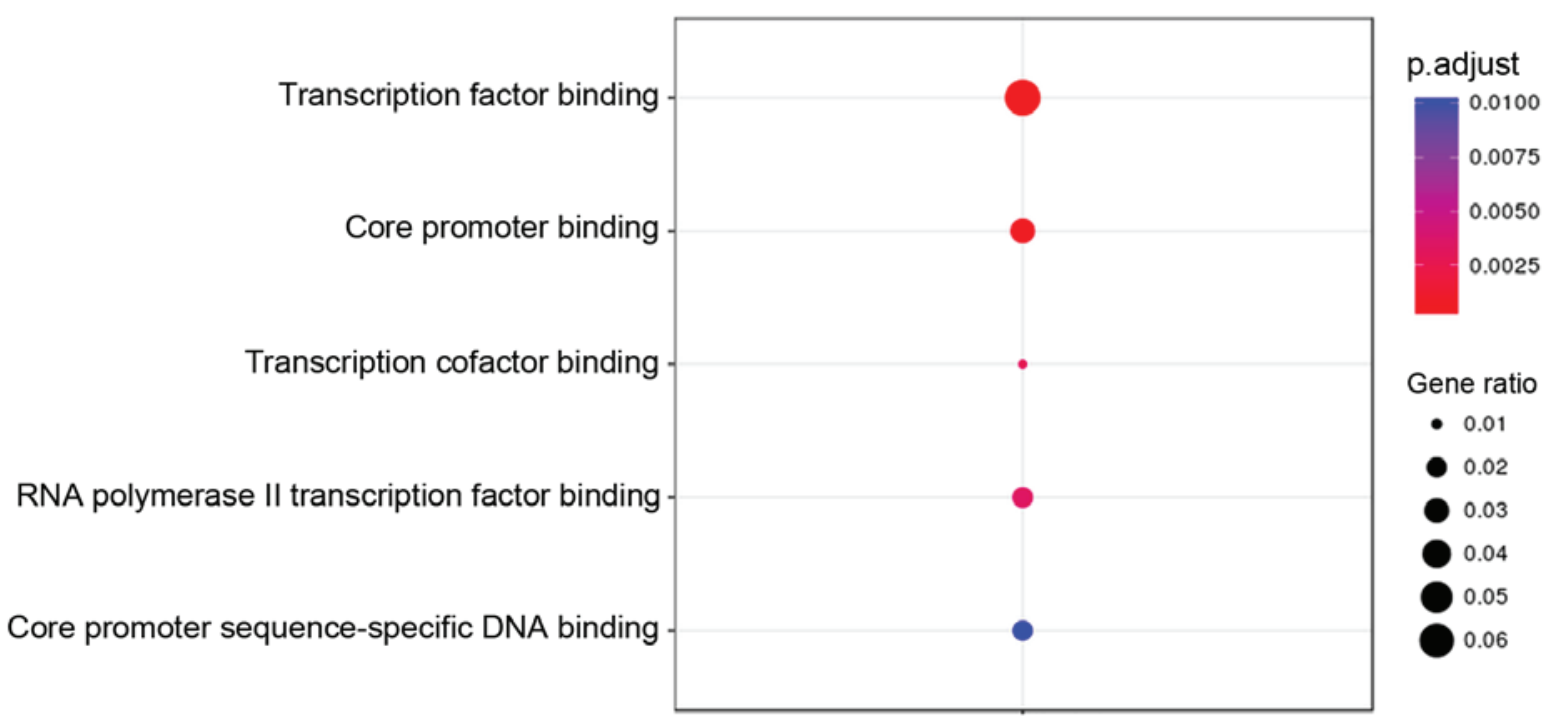

Figure 4. Gene oncology analysis of genes associated with hypomethylated CpGs in Alzheimer's disease subjects.

state (29). In the current study, it was identified that genes associated with hypermethylated $\mathrm{CpGs}$ participated in two Wnt-associated biological processes, including the Wnt signaling pathway and cell-cell signaling by Wnt. The Wnt signaling pathway is a vital signal transduction pathway involved in various biological activities. Increasing evidence suggested the protective effects of the Wnt pathway on synaptic modulation and cognitive processes in neurodegenerative diseases, implying the potential role of aberrant Wnt signaling in cognitive impairment $(30,31)$. Riise et al (32) also confirmed an aberrant Wnt signaling pathway in medial temporal lobe structures of neurodegenerative AD samples. Recent studies have further affirmed the potential role of the Wnt signaling pathway as a therapeutic target for the treatment of $\operatorname{AD}(33,34)$.
In the present study, hypomethylation was detected in genes involved in transcription factor binding and core promoter binding. Numerous studies have revealed the effects of DNA methylation on the transcription factor binding (35-37). Transcription factors serve important roles in the epigenetic regulation of gene transcription, while DNA methylation prohibits the recruitment of transcription factors, resulting in transcription suppression. A protein microarray-based approach observed that methylation-dependent transcription factor binding was a widespread phenomenon in biological processes (38). Methylated CpG sites are typically considered to reduce gene expression by preventing transcription factors from binding to promoter regions (38). Promoter site-specific $\mathrm{CpG}$ methylation is associated with various biological processes. Cyclical methylation alterations in promoter $\mathrm{CpGs}$ 
represent a critical event in achieving transcription (39). Pieper et al (40) also identified a tendency for hypomethylation in the tumor necrosis factor $\alpha$ (TNF- $\alpha$ ) core promoter region in neurodegenerative disorders, resulting in reduced binding of the transcription factors and suppressed TNF- $\alpha$ promoter activity.

The focus of the present study was the STG region of patients with late-onset AD. Increasing studies have already identified unique epigenetic signatures involved in the progression of AD in various brain regions. For instance, human AD case-cognitively normal control studies observed global hypomethylation in the entorhinal cortex (41) and in the temporal neocortex neuronal nuclei (42). Additionally, Bakulski et al (13) demonstrated widespread discordant DNA methylation in AD in the human frontal cortex. Chouliaras et al (43) identified a consistent decrease in global DNA methylation and hydroxymethylation in the hippocampus of AD subjects. Furthermore, the present study demonstrated a strong bias to hypermethylated alterations in the STG region in AD patients, implying different methylation changes across various brain regions.

In conclusion, the present study demonstrated significant and distinctive DNA methylation patterns in the STG region of patients with late-onset AD. Hypermethylation was mainly detected for genes regulating the cell cycle progression, while hypomethylation was identified in genes involved in transcription factor binding. These results suggested AD-associated epigenetic marks, potentially contributing to the understanding of underlying mechanisms involved in the epigenetic regulation of $\mathrm{AD}$ and the development of novel therapeutic targets for $\mathrm{AD}$.

\section{References}

1. Wilson RS, Segawa E, Boyle PA, Anagnos SE, Hizel LP and Bennett DA: The natural history of cognitive decline in Alzheimer's disease. Psychol Aging 27: 1008-1017, 2012.

2. Association As: 2017 Alzheimer's disease facts and figures. Alzheimers Dement 13: 325-37310, 2017.

3. Selkoe DJ: Translating cell biology into therapeutic advances in Alzheimer's disease. Nature 399 (6738 Suppl): A23-A31, 1999.

4. Migliore L and Coppedè F: Genetics, environmental factors and the emerging role of epigenetics in neurodegenerative diseases. Mutat Res 667: 82-97, 2009.

5. Iatrou A, Kenis G, Rutten BP, Lunnon $K$ and van den Hove DL: Epigenetic dysregulation of brainstem nuclei in the pathogenesis of Alzheimer's disease: Looking in the correct place at the right time? Cell Mol Life Sci 74: 509-523, 2017.

6. Gatz M, Reynolds CA, Fratiglioni L, Johansson B, Mortimer JA, Berg S, Fiske A and Pedersen NL: Role of genes and environments for explaining Alzheimer disease. Arch Gen Psychiatry 63 : 168-174, 2006.

7. Bird A: Perceptions of epigenetics. Nature 447: 396-398, 2007.

8. Jones PA and Takai D: The role of DNA methylation in mammalian epigenetics. Science 293: 1068-1070, 2001.

9. Ziller MJ, Gu H, Müller F, Donaghey J, Tsai LT, Kohlbacher O, De Jager PL, Rosen ED, Bennett DA, Bernstein BE, et al: Charting a dynamic DNA methylation landscape of the human genome. Nature 500: 477-481, 2013.

10. Smith ZD and Meissner A: DNA methylation: Roles in mammalian development. Nat Rev Genet 14: 204-220, 2013.

11. Wang J, Yu JT, Tan MS, Jiang T and Tan L: Epigenetic mechanisms in Alzheimer's disease: Implications for pathogenesis and therapy. Ageing Res Rev 12: 1024-1041, 2013.

12. Bollati V, Galimberti D, Pergoli L, Dalla Valle E, Barretta F, Cortini F, Scarpini E, Bertazzi PA and Baccarelli A: DNA methylation in repetitive elements and Alzheimer disease. Brain Behav Immun 25: 1078-1083, 2011.
13. Bakulski KM, Dolinoy DC, Sartor MA, Paulson HL, Konen JR, Lieberman AP, Albin RL, Hu H and Rozek LS: Genome-wide DNA methylation differences between late-onset Alzheimer's disease and cognitively normal controls in human frontal cortex. J Alzheimers Dis 29: 571-588, 2012.

14. Hannum G, Guinney J, Zhao L, Zhang L, Hughes G, Sadda S, Klotzle B, Bibikova M, Fan JB, Gao Y, et al: Genome-wide methylation profiles reveal quantitative views of human aging rates. Mol Cell 49: 359-367, 2013.

15. Bussière T, Gold G, Kövari E, Giannakopoulos P, Bouras C, Perl DP, Morrison JH and Hof PR: Stereologic analysis of neurofibrillary tangle formation in prefrontal cortex area 9 in aging and Alzheimer's disease. Neuroscience 117: 577-592, 2003.

16. von Gunten A, Kövari E, Rivara CB, Bouras C, Hof PR and Giannakopoulos P: Stereologic analysis of hippocampal Alzheimer's disease pathology in the oldest-old: Evidence for sparing of the entorhinal cortex and CA1 field. Exp Neurol 193: 198-206, 2005

17. Ginsberg SD, Hemby SE, Lee VM, Eberwine JH and Trojanowski JQ: Expression profile of transcripts in Alzheimer's disease tangle-bearing CA1 neurons. Ann Neurol 48: 77-87, 2000 .

18. Haroutunian V, Katsel P and Schmeidler J: Transcriptional vulnerability of brain regions in Alzheimer's disease and dementia. Neurobiol Aging 30: 561-573, 2009.

19. Watson CT, Roussos P, Garg P, Ho DJ, Azam N, Katsel PL, Haroutunian V and Sharp AJ: Genome-wide DNA methylation profiling in the superior temporal gyrus reveals epigenetic signatures associated with Alzheimer's disease. Genome Med 8: $5,2016$.

20. Huynh JL, Garg P, Thin TH, Yoo S, Dutta R, Trapp BD, Haroutunian V, Zhu J, Donovan MJ, Sharp AJ and Casaccia P: Epigenome-wide differences in pathology-free regions of multiple sclerosis-affected brains. Nat Neurosci 17: 121-130, 2014

21. Du P, Kibbe WA and Lin SM: lumi: A pipeline for processing illumina microarray. Bioinformatics 24: 1547-1548, 2008.

22. Teschendorff AE, Marabita F, Lechner M, Bartlett T, Tegner J, Gomez-Cabrero D and Beck S: A beta-mixture quantile normalization method for correcting probe design bias in Illumina Infinium 450 k DNA methylation data. Bioinformatics 29: 189-196, 2013.

23. Stadler MB, Murr R, Burger L, Ivanek R, Lienert F, Schöler A, van Nimwegen E, Wirbelauer C, Oakeley EJ, Gaidatzis D, et al: DNA-binding factors shape the mouse methylome at distal regulatory regions. Nature 480: 490-495, 2011.

24. Finer S, Mathews C, Lowe R, Smart M, Hillman S, Foo L, Sinha A, Williams D, Rakyan VK and Hitman GA: Maternal gestational diabetes is associated with genome-wide DNA methylation variation in placenta and cord blood of exposed offspring. Hum Mol Genet 24: 3021-3029, 2015.

25. Sturn A, Quackenbush J and Trajanoski Z: Genesis: Cluster analysis of microarray data. Bioinformatics 18: 207-218, 2002.

26. Millan MJ: The epigenetic dimension of Alzheimer's disease: Causal, consequence, or curiosity? Dialogues Clin Neurosci 16: 373-393, 2014.

27. Hebert LE, Bienias JL, Aggarwal NT, Wilson RS, Bennett DA, Shah RC and Evans DA: Change in risk of Alzheimer disease over time. Neurology 75: 786-791, 2010.

28. Hernandez DG, Nalls MA, Gibbs JR, Arepalli S, van der Brug M, Chong S, Moore M, Longo DL, Cookson MR, Traynor BJ and Singleton AB: Distinct DNA methylation changes highly correlated with chronological age in the human brain. Hum Mol Genet 20: 1164-1172, 2011.

29. Probst AV, Dunleavy E and Almouzni G: Epigenetic inheritance during the cell cycle. Nat Rev Mol Cell Biol 10: 192-206, 2009.

30. Ortiz-Matamoros A, Salcedo-Tello P, Avila-Muñoz E, Zepeda A and Arias C: Role of Wnt signaling in the control of adult hippocampal functioning in health and disease: Therapeutic implications. Curr Neuropharmacol 11: 465-476, 2013.

31. Arrázola MS, Silva-Alvarez C and Inestrosa NC: How the Wnt signaling pathway protects from neurodegeneration: The mitochondrial scenario. Front Cell Neurosci 9: 166, 2015.

32. Riise J, Plath N, Pakkenberg B and Parachikova A: Aberrant Wnt signaling pathway in medial temporal lobe structures of Alzheimer's disease. J Neural Transm (Vienna) 122: 1303-1318, 2015.

33. Sinha A, Tamboli RS, Seth B, Kanhed AM, Tiwari SK, Agarwal S, Nair S, Giridhar R, Chaturvedi RK and Yadav MR: Neuroprotective role of novel triazine derivatives by activating $\mathrm{Wnt} / \beta$ catenin signaling pathway in rodent models of alzheimer's disease. Mol Neurobiol 52: 638-652, 2015. 
34. del Pino J, Ramos E, Aguilera OM, Marco-Contelles J and Romero A: Wnt signaling pathway, a potential target for Alzheimer's disease treatment, is activated by a novel multitarget compound ASS234. CNS Neurosci Ther 20: 568-570, 2014.

35. Tenayuca J, Cousins K, Yang S and Zhang L: Computational modeling approach in probing the effects of cytosine methylation on the transcription factor binding to DNA. Curr Top Med Chem 17: 1778-1787, 2017.

36. Banovich NE, Lan X, McVicker G, van de Geijn B, Degner JF, Blischak JD, Roux J, Pritchard JK and Gilad Y: Methylation QTLs are associated with coordinated changes in transcription factor binding, histone modifications and gene expression levels. PLoS Genet 10: e1004663, 2014

37. Medvedeva YA, Khamis AM, Kulakovskiy IV, Ba-Alawi W, Bhuyan MS, Kawaji H, Lassmann T, Harbers M, Forrest AR and Bajic VB; FANTOM consortium: Effects of cytosine methylation on transcription factor binding sites. BMC Genomics 15: 119, 2014.

38. Hu S, Wan J, Su Y, Song Q, Zeng Y, Nguyen HN, Shin J, Cox E, Rho HS and Woodard C, et al: DNA methylation presents distinct binding sites for human transcription factors. Elife 2: e00726, 2013.

39. Métivier R, Gallais R, Tiffoche C, Le Péron C, Jurkowska RZ, Carmouche RP, Ibberson D, Barath P, Demay F and Reid G: Cyclical DNA methylation of a transcriptionally active promoter. Nature 452: 45-50, 2008.

40. Pieper HC, Evert BO, Kaut O, Riederer PF, Waha A and Wüllner U: Different methylation of the TNF-alpha promoter in cortex and substantia nigra: Implications for selective neuronal vulnerability. Neurobiol Dis 32: 521-527, 2008.
41. Mastroeni D, Grover A, Delvaux E, Whiteside C, Coleman PD and Rogers J: Epigenetic changes in Alzheimer's disease: Decrements in DNA methylation. Neurobiol Aging 31: 2025-2037, 2010.

42. Mastroeni D, McKee A, Grover A, Rogers J and Coleman PD: Epigenetic differences in cortical neurons from a pair of monozygotic twins discordant for Alzheimer's disease. PLoS One 4: e6617, 2009.

43. Chouliaras L, Mastroeni D, Delvaux E, Grover A, Kenis G, Hof PR, Steinbusch HW, Coleman PD, Rutten BP and van den Hove DL: Consistent decrease in global DNA methylation and hydroxymethylation in the hippocampus of Alzheimer's disease patients. Neurobiol Aging 34: 2091-2099, 2013.

(c) (1) 9 This work is licensed under a Creative Commons Attribution-NonCommercial-NoDerivatives 4.0 International (CC BY-NC-ND 4.0) License. 